\title{
Comparison of Open-Source Electronic Health Record Systems Based on Functional and User Performance Criteria
}

\author{
Saptarshi Purkayastha ${ }^{1}$, Roshini Allam ${ }^{1}$, Pallavi Maity ${ }^{1}$, Judy W. Gichoya ${ }^{2}$ \\ 'Department of BioHealth Informatics, Indiana University-Purdue University Indianapolis, Indianapolis, IN, USA \\ ${ }^{2}$ Dotter Institute, Oregon Health and Science University, Portland, OR, USA
}

Objectives: Open-source Electronic Health Record (EHR) systems have gained importance. The main aim of our research is to guide organizational choice by comparing the features, functionality, and user-facing system performance of the five most popular open-source EHR systems. Methods: We performed qualitative content analysis with a directed approach on recently published literature (2012-2017) to develop an integrated set of criteria to compare the EHR systems. The functional criteria are an integration of the literature, meaningful use criteria, and the Institute of Medicine's functional requirements of EHR, whereas the user-facing system performance is based on the time required to perform basic tasks within the EHR system. Results: Based on the Alexa web ranking and Google Trends, the five most popular EHR systems at the time of our study were OSHERA VistA, GNU Health, the Open Medical Record System (OpenMRS), Open Electronic Medical Record (OpenEMR), and OpenEHR. We also found the trends in popularity of the EHR systems and the locations where they were more popular than others. OpenEMR met all the 32 functional criteria, OSHERA VistA met 28, OpenMRS met 12 fully and 11 partially, OpenEHR-based EHR met 10 fully and 3 partially, and GNU Health met the least with only 10 criteria fully and 2 partially. Conclusions: Based on our functional criteria, OpenEMR is the most promising EHR system, closely followed by VistA. With regards to user-facing system performance, OpenMRS has superior performance in comparison to OpenEMR.

Keywords: Electronic Health Record, Decision Support Systems, Clinical, Medical Order Entry Systems, Electronic Prescribing

Submitted: January 21, 2019

Revised: February 22, 2019

Accepted: March 13, 2019

\section{Corresponding Author}

Saptarshi Purkayastha

Department of BioHealth Informatics, Indiana University-Purdue University Indianapolis, 719 Indiana Ave, WK 119, Indianapolis, IN, USA. Tel: +1-317-274-0439, E-mail: saptpurk@iupui.edu (https:// orcid.org/0000-0003-3625-534X)

This is an Open Access article distributed under the terms of the Creative Commons Attribution Non-Commercial License (http://creativecommons.org/licenses/by$\mathrm{nc} / 4.0 /$ ) which permits unrestricted non-commercial use, distribution, and reproduction in any medium, provided the original work is properly cited.

(c) 2019 The Korean Society of Medical Informatics

\section{Introduction}

Open-source software (OSS) is computer software with a licensing provision that enables the users to modify, utilize, and distribute unmodified or modified versions [1]. The free open-source software (FOSS) is also known as libre and open-source software (FLOSS), libre software, free software, and open-source software. In recent decades, the opensource business model has evolved, and FOSS is now more than just cost-free software. Because source code is available, FOSS has developed into many different distribution models [2]. OSS has several technical benefits, such as reliability, security, quality, good performance, flexibility of use, tester 
and user base, compatibility, and harmonization [3]. FOSS in healthcare is different because of the inherent need for domain-specific experts in the community. From 1999 to 2001, the healthcare industry saw the start of many OSS projects [4]. Several factors have contributed to the success of software products in healthcare. These include the existence of a user community and a developer community, regular release cycles, modular architecture, documentation of software, and support services [4].

One of the most significant benefits of OSS in the healthcare industry is that FOSS is easy to obtain as it can be downloaded easily from repositories. There are no restrictive licensing terms regarding distribution, derivative works, or modifications, and there are no software licensing fees. Open-source software is vendor neutral, which means that organizations or individuals do not suffer from vendor lock-in. Although there are numerous other benefits of cost savings and flexibility, opportunities to innovate while remaining independent from vendors is more applicable to healthcare [3].

Challenges of OSS in healthcare are organizational in nature, such as long-term support organization, funding and ownership for EHR certification, open-source business models, medical informatics and domain specific issues, functionality-based limitations, usability issues, challenges in resource-restricted environments, and challenges related human factors [5]. The main objective of this work is to guide organizational choice to select an open-source system for implementation by comparing the features and functionality of the five most popular open-source Electronic Health Record (EHR) systems using an integrated set of criteria. It might also provide useful insight for open-source developers to identify the functional requirements that are missing but required by clinical users. The user performance comparison results may be used by EHR developers to analyze and improve the usability of their products for basic tasks, such as patient search, ordering, viewing patient records, and so forth.

\section{Methods}

\section{Information Source and Search}

Relevant articles on open-source EHR systems were identified primarily using Scopus. We also used other databases, such as PubMed and IEEE Xplore, to ensure that we would not leave out any publications that are not indexed by Scopus. We searched publications from 2012 to 2017 to identify criteria to compare open-source EHR systems. Only Eng- lish language articles were selected. The search terms were looked up in their titles and abstracts.

\section{Study Selection, Data Collection Process, and Data Items}

The next step was a detailed examination of papers and exclusion of irrelevant papers. We included articles that discussed 'open-source electronic health record systems' AND 'comparison' OR 'adoption' OR 'utilization' OR 'analysis' OR 'application'. Figure 1 presents the PRISMA flowchart outlining the process for systematic review. Only full-text available articles were selected. Finally, we included a total of 25 articles.

\section{Literature Review}

A study conducted by Zaidan et al. [6], evaluated various open-source EHR systems using multi-criteria decisionmaking of AHP-TOPSIS, which is complicated and requires an enterprise-wide understanding of the concept of an ideal solution. To assess OSS EHR functionality, we considered the following eight parameters: health information and data, results management, order entry and management, decision support, electronic communication and connectivity, patient support, administrative processes, and reporting and population health management. This is based on the Institute of Medicine (IOM), renamed as the National Academy of Medicine's eight-core features of an EHR system.

Goldwater et al. [7] studied the use of open-source EHR systems in chronic disease management. The study showed the positive impact of an open-source EHR system in im-

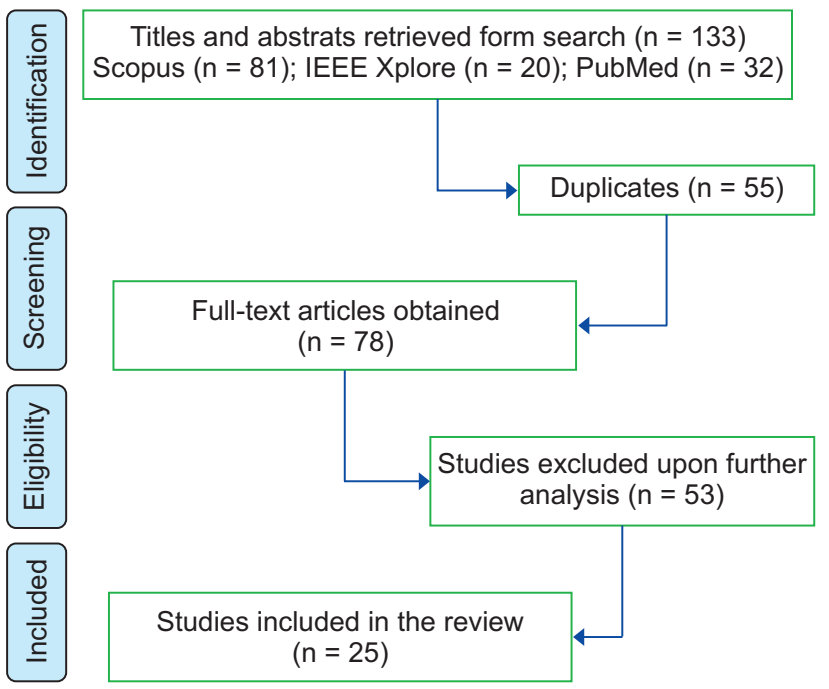

Figure 1. PRISMA flowchart outlining the process of systematic review. 
proving diabetes and tuberculosis management and controlling hypertension. They identified six essential elements that should be available in an EHR system to support chronic disease management. These elements include modern clinical information services, systematic decision support systems, self-management support for patients, and links to community resources [7]. Meaningful use criteria include clinical decision support and availability of patient-specific resources; therefore, we included them in our integrated functional criteria [8].

Based on the results of the qualitative content analysis we selected meaningful use criteria and the eight-core functionalities from the IOM, finally totaling 32 criteria to compare the features and functionalities of various health FOSS. We adopted a set of performance criteria from the study conducted by de Abajo and Ballestero [9] to compare OpenMRS, OpenEMR, and VistA. Along with the literature, we thoroughly reviewed all the software documentation on the websites of the five EHR systems.

\section{Comparison Criteria}

The study was conducted as follows. The open-source EHR systems were compared using a set of performancemeasuring parameters by deploying the software on a local machine. Then they were compared in terms of meaningful use criteria and the IOM core functionalities. Figure 2 shows an overview of the methodology.

1) Identification and selection of open-source EHR systems The open-source EHR systems were selected in two steps. The top five EHR systems were selected based on their global website ranking and identification of the reach and popularity of open-source EHR systems over time. Alexa is a global ranking system that utilizes web traffic data to compile rankings. From the 15 listed open-source EHR systems (See Table 1), VistA, GNU Health, OpenMRS, OpenEMR, and OpenEHR were selected based on their global rankings retrieved from Alexa rank. HOSxP changed its license in 2015, and we excluded it from our study due to its focus on a subscription-based hosted EHR. These five EHR systems

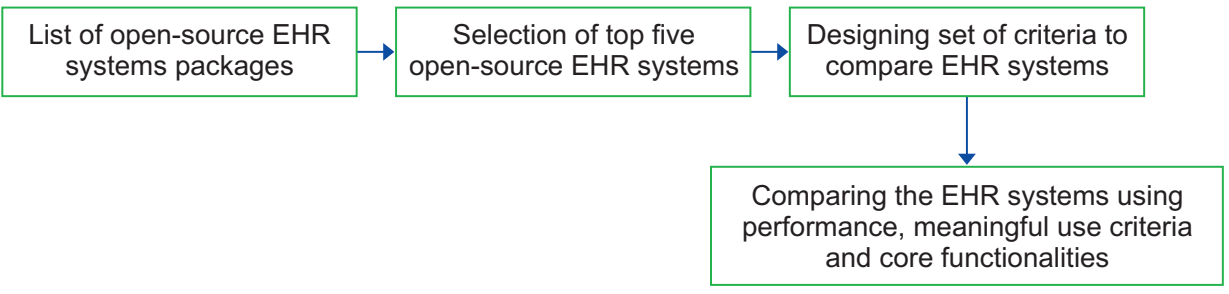

Figure 2. Flowchart illustrating the methods of the current study. EHR: Electronic Health Record.

Table 1. List of open-source EHR systems along with the official website and its global rank

\begin{tabular}{llc}
\hline \multicolumn{1}{c}{ EHR system } & \multicolumn{1}{c}{ Link } & Global rank \\
\hline VistA (OSHERA) & http://www.ehealth.va.gov & 4,052 \\
GNU Health & http://health.gnu.org & 6,355 \\
OpenMRS & https://openmrs.org & 187,710 \\
OpenEMR & http://www.open-emr.org & 381,856 \\
HOSxP & http://hosxp.net/joomla25 & 407,971 \\
OpenEHR & http://openehr.org & $1,199,683$ \\
OSCAR & http://oscarmcmaster.org & $1,027,913$ \\
CARE2X system & http://care2x.org & $4,314,882$ \\
Hospital OS & http://www.hospital-os.com & $5,701,901$ \\
FreeMed & http://freemedsoftware.org & $4,496,417$ \\
MedinTux & http://www.medintux.org & $5,655,226$ \\
GNUMed & http://wiki.gnumed.de/bin/view/Gnumed & $2,567,684$ \\
ClearHealth & http://clear-health.com & $5,701,006$ \\
THIRRA & http://thirra.primacare.org.my & Nttp://www.ictedge.org/projects/zeprs global rank \\
ZEPRS & & No global rank \\
\hline
\end{tabular}

EHR: Electronic Health Record. 
vary greatly regarding their audience and the type of health facility that they target. However, we compared the clinical features that should be present in all EHR systems, irrespective of whether they are implemented in a primary care clinic, community hospital, or a tertiary hospital, even though the sophistication of those functionalities might differ based on needs. OpenEHR is a standard, a set of archetypes that are implemented in EHR system products. OSEHRA VistA and GNU Health are hospital information systems. OpenEMR and OpenMRS are EHR systems or platforms, respectively, but those are details that are common across proprietary EHR systems, which market their products to different audiences and focus on certain types of clinical settings. A Google Trends comparison of the five selected EHR systems from August 2017 to August 2018 is shown in Figure 3.

Figure 4 shows the regional popularity of each of the five EHR systems. It is interesting to note that OpenEHR is clearly more popular in Northern European countries, Scandinavia, Brazil, and Australia than the rest of the world. GNU Health has some interest in China, the US, Argentina, Germany, and Spain. OpenEMR and OpenMRS have more global reach, and this can also be identified by consideration of the global implementation map of these EHR systems. OpenMRS is clearly very popular and widely implemented in Africa, India, and South East Asia. OpenEMR has implementations in India, the United States, Brazil, the UK, and South Korea. VistA is primarily used by the Veterans Affairs (VHA) in the United States, with some custom implementations and distributions of it in other places.

\section{2) Evaluation}

The evaluation in our study was carried out in two main phase, one by deploying the software and comparing the EHR systems using the standard set of criteria, and subsequently by comparing the performance parameters.

(i) Deploying each open-source EHR software: Documentation available on respective EHR system official websites were used for deployment.

(ii) Establishing criteria to evaluate and compare: Based on the literature review summarized above, using qualitative content analysis, we developed an evaluation criterion extracted from the meaningful use criteria and eight-
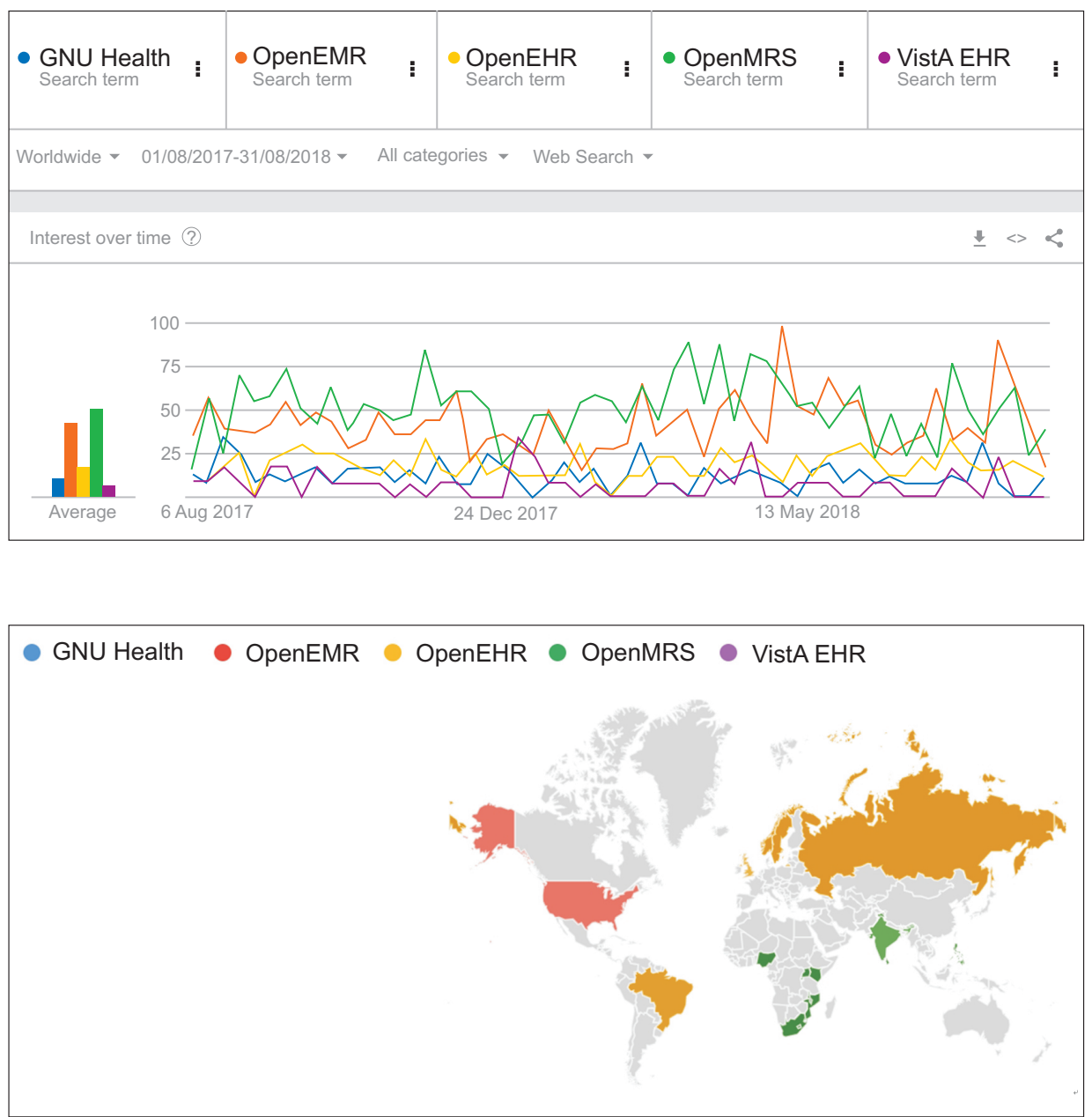

Figure 3. Comparison of interest over time (August 2017 to August 2018) for the five EHR systems. EMR: Electronic Medical Record, EHR: Electronic Health Record, MRS: Medical Record System. 
core functionalities listed by the IOM [10].

\section{3) Performance criteria}

The selected parameters of user performance criteria include initial CPU use, startup time of the server, shutdown time of server, login time and CPU use, patient search time and $\mathrm{CPU}$ use, time for creating new patient and CPU use, time for change of patient and CPU use, average navigation time for the main menu and CPU use, logout time and CPU use. These performance criteria were implemented on a machine with a 64-bit Windows 10 operating system and 8 GB RAM.

\section{Results}

The interoperability comparison focused on criteria based on meaningful use presented in Table 2.

\section{Transition of Care and Clinical Information Reconciliation}

ZH Healthcare incorporated a mechanism to upload, process, display, and incorporate CCDA into OpenEMR. In OSEHRA VistA, this criterion is fulfilled by popHealth, which is an open-source quality measure reference implementation. In OpenEMR clinical information, the reconciliation criterion is fulfilled by the $\mathrm{ZH}$ Healthcare coordination module.
OpenMRS stores all of a patient's details, visits, encounters, and observations.

\section{Patient-Specific Education Resources and e-Prescribing} This is available only in OpenEMR and OSEHRA VistA. In OpenEMR, this feature is available under the patient support tab in the user interface, and in OSEHRA VistA, this criterion is fulfilled by infobuttons. In OpenEMR (see Figure 5), eprescribing can be done in three ways: Newcrop (a subscription service integrated with OpenEMR), AllScripts (it does not have fees, but it focuses on the US phamacy networks), and Weno (another US-centric, free eRx routing service). On the other hand, GNU Health (see Figure 6) has a very local formulary-focused workflow, where all drugs have to be entered manually, and prescriptions are made from those. In OpenEHR, this criterion is fulfilled under a locationindependent medication management feature. In the case of VistA, it has a separate e-prescribing module.

\section{Incorporation and Transmission of Electronic Laboratory Tests}

In OpenEMR, this has been supported by the ZH Healthcare (an EHR vendor) laboratory module. In OpenMRS and GNU Health, laboratory results are stored, but not in accordance with the HL7 implementation guide. In OSEHRA

Table 2. Findings of interoperability-focused criteria

\begin{tabular}{|c|c|c|c|c|c|}
\hline Interoperability-focused criteria & OpenEMR & OpenEHR & OpenMRS & GNU Health & OSHERA VistA \\
\hline Transition of care & Yes & None & Partial & None & Yes \\
\hline Clinical information reconciliation & Yes & None & Yes & Yes & Yes \\
\hline Clinical decision support & Yes & Partial & Partial & Partial & Yes \\
\hline Patient-specific resources & Yes & None & None & None & Yes \\
\hline E-prescribing & Yes & Yes & None & Yes & Yes \\
\hline Incorporation of laboratory tests and values/results & Yes & Yes & Partial & Partial & Yes \\
\hline $\begin{array}{l}\text { Transmission of electronic laboratory tests and } \\
\text { values/results to ambulatory providers }\end{array}$ & Yes & Yes & Yes & Yes & Yes \\
\hline Data portability & Yes & None & Partial & None & Yes \\
\hline Clinical quality measures & Yes & Partial & Partial & Yes & Yes \\
\hline Viewing, downloading, and transmission to 3rd party & Yes & None & Partial & None & Yes \\
\hline Clinical summaries & Yes & Partial & Yes & None & Yes \\
\hline Transmission to immunization registries & Yes & None & None & None & None \\
\hline $\begin{array}{l}\text { Transmission of syndromic surveillance to } \\
\text { public health agencies }\end{array}$ & Yes & None & Partial & None & Yes \\
\hline Transmission of lab results to public health agencies & Yes & None & Partial & None & Yes \\
\hline Optional transmission to cancer registries & Yes & None & None & None & Yes \\
\hline
\end{tabular}

EMR: Electronic Medical Record, EHR: Electronic Health Record, MRS: Medical Record System. 


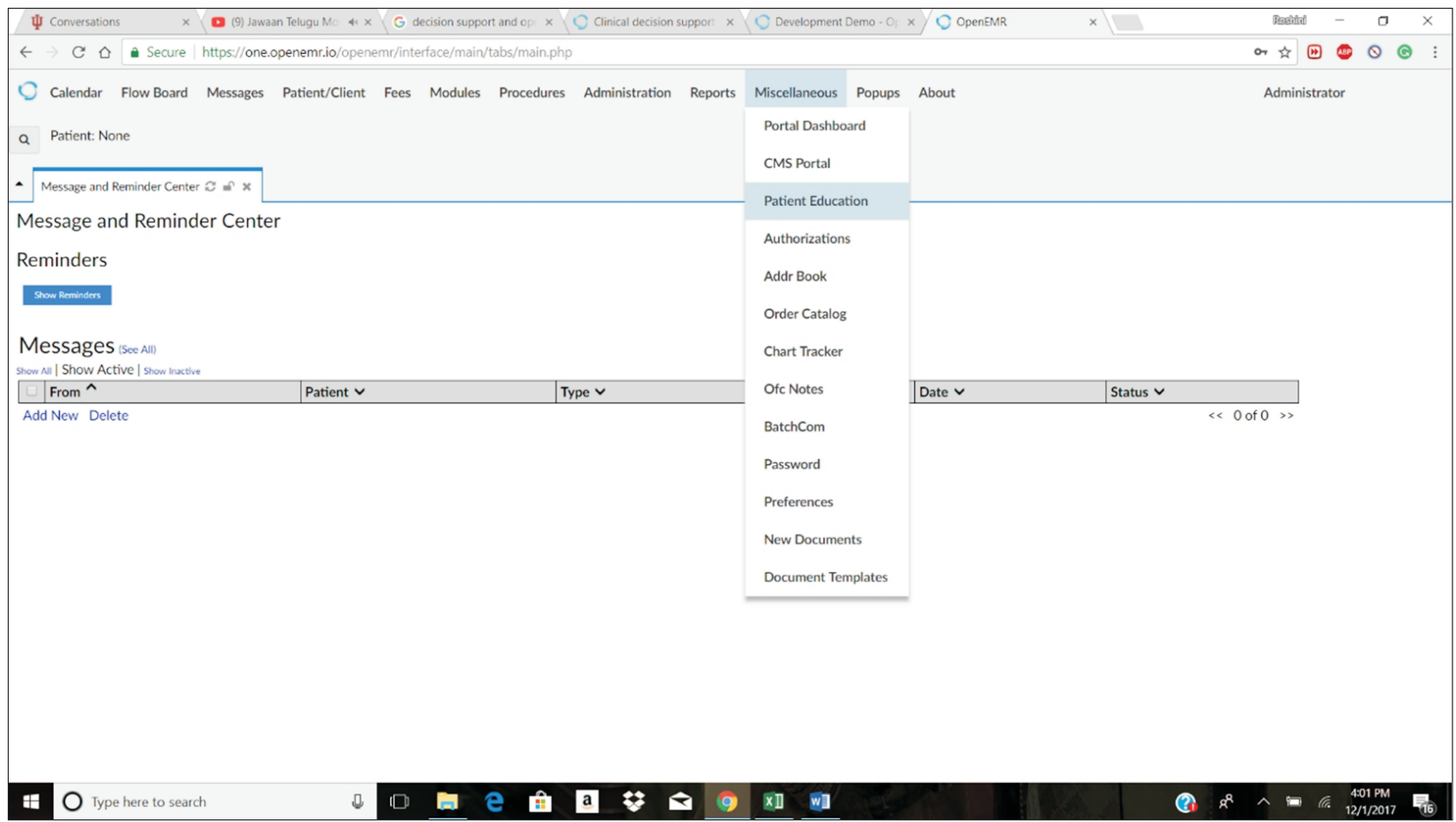

Figure 5. Patient education with messages and reminders in Open Electronic Medical Record (OpenEMR).

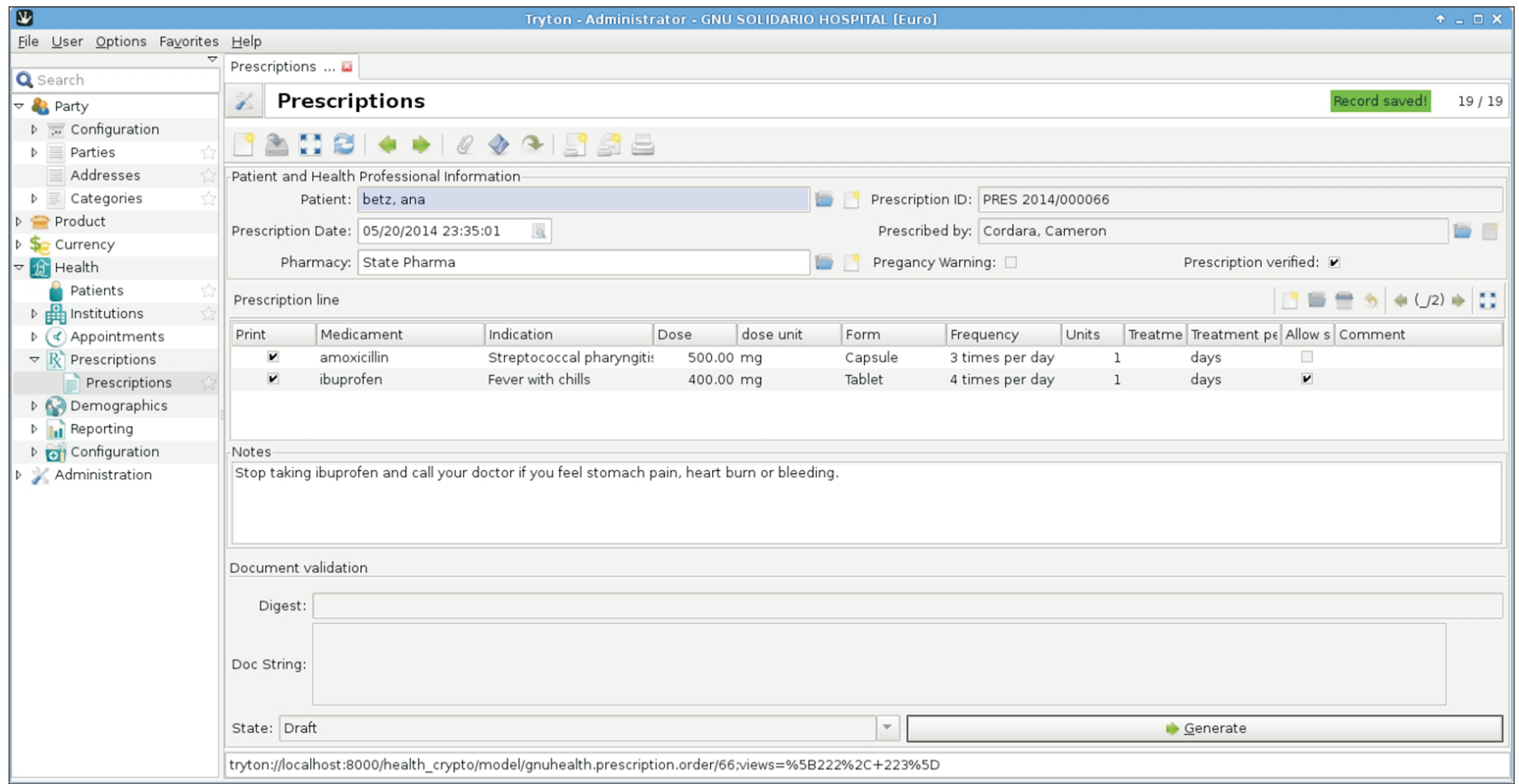

Figure 6. Generation of e-prescriptions in GNU Health.

VistA, this criterion is fulfilled by the VistA laboratory module. Transmission of electronic laboratory tests is possible in all the selected systems. In openEHR, laboratory results can be transmitted to other EHR systems. In the case of VistA, this criterion is fulfilled by the VistA laboratory module.

\section{Data Portability and Clinical Quality Measures}

In openEHR, this is supported by the ZH Healthcare care coordination module. OpenMRS exports according to the Clinical Document Architecture (CDA) standard, not the CCDA (a US-specific standard Consolidated CDA) standard. In VistA, data portability is fulfilled by a separate but 
related product called popHealth. OpenEMR supports capture, calculation, import, export, and electronic submission of clinical quality measures. OpenMRS, GNU Health, and OpenEHR calculate quality indicators only. In VistA, the CQM criterion is fulfilled by popHealth.

A comparison of the privacy- and security-focused meaningful use criteria are presented in Table 3.

\section{Authentication, Access Control, and Authorization}

OpenEMR supports security measures, such as the authentication mechanism, access control, and authentication. In OpenEHR, this provided by Inidis, which is one of the OpenEHR partners. GNU Health has a central authentication system, and access control is fulfilled by security features in GNU Health. VistA has a strong access management architecture.

\section{Automatic Log-Off and Integrity}

In OpenEMR, the automatic log-off feature was tested and confirmed by Jones et al. [11]. In VistA, the auto log-out feature can be enabled by applying a few settings on the VA user profile. Integrity is implemented in OpenEMR as part of the Certification Commission for Healthcare Information Technology (CCHIT) requirements. In OpenMRS, the integrity of data is ensured by the data integrity module. In OpenEHR, integrity is provided by the versioning model specified in the change_control package.

\section{Order Entry/Order Management and Decision Support} OpenEHR, OpenEMR, and VistA allow computerized provider order entry. In OpenEHR, clinical decision support is delivered through guideline definition language. GNU Health also uses the World Health Organization essential list of medicines. In VistA, decision support is supported by infobuttons.

\section{Electronic Communication, Connectivity, Patient Support and Reporting for Population Health}

Electronic communication between providers is available only in OpenEMR, OpenMRS, and OSEHRA VistA. In

Table 3. Privacy- and security-focused meaningful use criteria

\begin{tabular}{lccccc}
\hline \multicolumn{1}{c}{ Privacy- and security-focused } & OpenEMR & OpenEHR & OpenMRS & GNU Health & OSHERA VistA \\
\hline Authentication, access control, and authorization & Yes & Yes & Yes & Yes & Yes \\
Auditable events and tamper-resistance & Yes & None & Yes & None & None \\
Audit reports & Yes & Yes & Partial & None & Yes \\
Amendments & Yes & None & None & None & Yes \\
Automatic log-off & Yes & None & None & None & Yes \\
Emergency access & Yes & None & Yes & None & Yes \\
End-user device encryption & Yes & None & Partial & None & None \\
Integrity & Yes & Yes & Yes & Yes & Yes \\
Optional accounting of disclosures & Yes & None & None & None & None \\
\hline
\end{tabular}

EMR: Electronic Medical Record, EHR: Electronic Health Record, MRS: Medical Record System.

Table 4. Institute of Medicine's core features criteria

\begin{tabular}{lccccc}
\hline \multicolumn{1}{c}{ Functionality } & OpenEMR & OpenEHR & OpenMRS & GNU Health & OSHERA VistA \\
\hline Health information and data & Yes & Yes & Yes & Yes & Yes \\
Results management & Yes & Yes & Yes & Yes & Yes \\
Order entry/order management & Yes & Yes & None & None & Yes \\
Decision support & Yes & Yes & Yes & Yes & Yes \\
Electronic communication and connectivity & Yes & None & Yes & No & Yes \\
Patient support & Yes & None & None & None & Yes \\
Administrative processes & Yes & Yes & Yes & Yes & Yes \\
Reporting and population health management & Yes & None & Partial & None & Yes \\
\hline
\end{tabular}

EMR: Electronic Medical Record, EHR: Electronic Health Record, MRS: Medical Record System. 
OpenEMR, patient-specific resources can be found under the patient support tab in the user interface. In VistA, patient-specific resources are supported by infobuttons. OpenEMR reports immunization information, syndromic surveillance, and cancer cases to public health agencies. Reporting of population health is done under the notifiable condition detector module. Table 4 compare the EHRs based on IOM's feature set.

\section{Discussion}

In this study, we compared the selected systems using the integrated criteria that were developed. Although a few studies have compared the EHR systems [12-14] that use a few criteria based on meaningful use, none of them have considered all the criteria listed by the Office of the National Coordinators for Health IT (ONC).

\section{Comparison with Meaningful Use Criteria}

In the context of health information technology, meaningful use is defined as the minimum US standard required for using EHR systems and for exchanging information between providers, between providers and insurance companies, and between providers and patients. While we acknowledge that other countries, particularly low- and middle-income countries $[15,16]$ might not legislatively require all of these features, their importance in all clinical settings cannot be ignored. Except for OpenEMR, none of the other selected systems have been verified for ONC's meaningful use criteria, though OSEHRA VistA has most of the criteria fulfilled, and some vendor distributions of VistA have been verified.

Limitation: More than 15 open-source EHR systems are available, but because of time constraints, we compared only the five most popular EHR systems.

\section{Core Functionalities}

OpenEMR and OSEHRA VistA have all the eight-core functionalities, while other systems are lacking a few. Health information and data, result management, decision support, and administrative processes are the functionalities available in all the selected EHR systems.

Limitation: Because of the unavailability of web-based demo databases for GNU Health, VistA, and OpenEHR, we had to deploy locally based on publicly available configuration documentation. We acknowledge that some vendors might deploy these systems differently. Also, certain EHR systems are specially or purposefully built for epidemics or disaster situations [17]. We ignore these as edge cases, and only consider general-purpose EHR systems.

\section{Performance}

The top two performing systems were OpenMRS and OpenEMR. The GNU Health, VistA, and OpenEHR-based systems were more complex and required many customiza-

Table 5. User performance criteria

\begin{tabular}{lcc}
\hline \multicolumn{1}{c}{ Criteria } & OpenEMR & OpenMRS \\
\hline Mean initial CPU use (\%) & 29.16 & 62.88 \\
The startup time of the application server (min) & 2.32 & 2.46 \\
The shutdown time of the application server (sec) & 58 & 12 \\
Mean login - CPU use (\%) & 11.48 & 11.2 \\
Mean login time (sec) & 2.282 & 1.349 \\
Mean patient search time (sec) & 2.86 & 2.97 \\
Mean patient search - CPU use (\%) & 16.6 & 28.42 \\
Mean CPU use for creating new patient (\%) & 16.72 & 2.056 \\
Mean time for creating new patient (sec) & 3.236 & 25.64 \\
Mean CPU use for changing patient (\%) & 24.9 & 3.18 \\
Mean time for changing patient (sec) & 6.3 & 829.4 \\
Mean navigation time for the main menu (ms) & 3,236 & 5.2 \\
Mean CPU use for navigation of the main menu (\%) & 6.66 & 782.8 \\
Mean logout time (ms) & 1,246 & 2.14 \\
Mean logout CPU use (\%) & 6.56 & \\
\hline
\end{tabular}

EMR: Electronic Medical Record, MRS: Medical Record System. 
tions before we could execute the user performance tasks. OpenMRS was found to be faster than OpenEMR. The CPU utilization is also higher with OpenEMR, as shown in Table 5. Limitation: The performance analysis was done on a single system, and this may differ from the performance of a system in a real-world setting. However, our goal was to test user performance on the most regular EHR tasks.

\section{Documentation}

OpenEMR, OSEHRA VistA, OpenMRS, and GNU Health have good documentation. OSEHRA VistA has a very detailed package list including their descriptions and implementation guide.

Limitation: The documentation for OpenEHR is very poor, which forced us to rely on other web resources and articles to support the availability of features.

\section{Conclusion}

Investigating available open-source EHR systems is necessary, but few studies have compared the existing opensource EHR systems. These studies on available open-source EHR systems are not exhaustive, especially on security and interoperability. In this study, the top five globally ranked EHR systems were compared using integrated criteria that we developed. Based on our findings, in terms of functionality, OpenEMR is the most promising EHR system, closely followed by VistA. Other systems were found to be lacking in a US-specific functional requirement, but also in some security and interoperability requirements. Regarding performance on the most common user tasks, OpenMRS achieves superior performance in comparison to OpenEMR.

\section{Conflict of Interest}

No potential conflict of interest relevant to this article was reported.

\section{ORCID}

Saptarshi Purkayastha (http://orcid.org/0000-0003-3625-534X)

Roshini Allam (http://orcid.org/0000-0002-2739-0913)

Pallavi Maity (http://orcid.org/0000-0001-5936-3226)

Judy W. Gichoya (http://orcid.org/0000-0002-1097-316X)

\section{References}

1. Bhandari G, Snowdon A. Adoption of open source software in healthcare. In: Phillips-Wren G, Jain LC, Naka- matsu K, et al., editors. Advances in intelligent decision technologies. Heidelberg: Springer; 2010. p. 365-72.

2. Saebi T, Foss NJ. Business models for open innovation: matching heterogeneous open innovation strategies with business model dimensions. Eur Manag J 2015;33(3):201-13.

3. Morgan L, Finnegan P. Benefits and drawbacks of open source software: an exploratory study of secondary software firms. In: Feller J, Fitzgerald B, Scacchi W, et al., editors. Open source development, adoption and innovation. Boston (MA): Springer; 2007. p. 307-12.

4. Reynolds CJ, Wyatt JC. Open source, open standards, and health care information systems. J Med Internet Res 2011;13(1):e24.

5. Ratib O, Rosset A, Heuberger J. Open Source software and social networks: disruptive alternatives for medical imaging. Eur J Radiol 2011;78(2):259-65.

6. Zaidan AA, Zaidan BB, Al-Haiqi A, Kiah ML, Hussain $M$, Abdulnabi M. Evaluation and selection of opensource EMR software packages based on integrated AHP and TOPSIS. J Biomed Inform 2015;53:390-404.

7. Goldwater JC, Kwon NJ, Nathanson A, Muckle AE, Brown A, Cornejo K. Open source electronic health records and chronic disease management. J Am Med Inform Assoc 2014;21(e1):e50-4.

8. Blumenthal D, Tavenner M. The "meaningful use" regulation for electronic health records. $\mathrm{N}$ Engl J Med 2010;363(6):501-4.

9. de Abajo BS, Ballestero AL. Overview of the most important open source software: analysis of the benefits of OpenMRS, OpenEMR, and VistA. In: Rodrigues JJ, de la Torre-Diez I, de Abajo BS, et al., editors. Telemedicine and e-health services, policies, and applications: advancements and developments. Hershey (PA): IGI Global; 2012. p. 315-46.

10. Tripathi M. EHR evolution: policy and legislation forces changing the EHR. J AHIMA 2012;83(10):24-9.

11. Jones B, Yuan X, Nuakoh E, Ibrahim K. Survey of open source health information systems. Health Inform 2014;3(1):23-31.

12. Karopka T, Schmuhl H, Demski H. Free/Libre open source software in health care: a review. Healthc Inform Res 2014;20(1):11-22.

13. Kiah ML, Haiqi A, Zaidan BB, Zaidan AA. Open source EMR software: profiling, insights and hands-on analysis. Comput Methods Programs Biomed 2014;117(2):36082.

14. Kashfi H. An openEHR-based clinical decision sup- 
port system: a case study. Stud Health Technol Inform 2009;150:348.

15. Syzdykova A, Malta A, Zolfo M, Diro E, Oliveira JL. Open-source electronic health record systems for lowresource settings: systematic review. JMIR Med Inform 2017;5(4):e44.

16. Alsaffar M, Yellowlees P, Odor A, Hogarth M. The state of open source electronic health record projects: a software anthropology study. JMIR Med Inform 2017;5(1):e6.

17. Oza S, Jazayeri D, Teich JM, Ball E, Nankubuge PA, Rwebembera J, et al. Development and deployment of the OpenMRS-Ebola electronic health record system for an Ebola treatment center in Sierra Leone. J Med Internet Res 2017;19(8):e294. 